\title{
The Mixed-Features Specifier of Major Depressive Disorder in DSM-5: Is It Practical?
}

\author{
Young-Min Park $\bowtie$ \\ Department of Psychiatry, Ilsan Paik Hospital, Inje University College of Medicine, Goyang, Republic of Korea
}

DSM-5 is the recent update of DSM IV-TR, and currently there is considerable controversy about the mixed-features specifier of major depressive disorder (MDD) for mixed depression in DSM-5. This mixed-features specifier consists of a major depressive episode and modified (hypo)manic criteria that were based on criteria B of bipolar disorder. The (hypo) manic criteria of this specifier and bipolar disorder are very similar to each other, although the former deleted distractibility and psychomotor agitation, and added elevated and expansive mood (in criteria A of bipolar disorder). These changes mean that the paradigm has shifted from a categorical concept into a dimensional or spectrum concept in mood disorder. However, some investigators are still criticizing the mixed-features specifier of MDD. They consider that these DSM- 5 criteria have a weak scientific basis and do not identify a large proportion of the possible mixed depressive states. ${ }^{1}$ Some authors have referred to these DSM- 5 criteria as 'a farewell to mixed depression' or 'the miracle that never happens. ${ }^{1,2}$

One of the reasons that authors have been criticizing DSM5 is that euphoria and grandiosity with MDD do not coexist commonly in clinical practice. ${ }^{3}$ Another reason is that DSM-5 excludes the core symptoms of mixed depression such as psychomotor agitation, irritability, and distractibility (so-called overlapping symptoms) because these symptoms may overlap with a major depressive episode. However, a recent study found that large proportions of patients presented with distractibility (59.2\%), irritability (57.3\%), and psychomotor agitation (36.5\%) in mixed depression, whereas only $18 \%$ and $6.6 \%$ presented with euphoria and grandiosity, respectively. ${ }^{4}$ Another study also found that agitation, marked irritability, and mood reactivity were common symptoms in mixed depression. ${ }^{5}$ Thus, adding overlapping or nonspecific symptoms to the criteria

$\triangle$ Correspondence: Young-Min Park, MD, PhD

Department of Psychiatry, Ilsan Paik Hospital, Inje University College of Medicine, 170 Juhwa-ro, Ilsanseo-gu, Goyang 10380, Republic of Korea

Tel: +82-31-910-7260, Fax: +82-31-910-7268, E-mail: medipark@hanmail.net

(c) This is an Open Access article distributed under the terms of the Creative Commons Attribution Non-Commercial License (https://creativecommons.org/licenses/by$\mathrm{nc} / 4.0$ ) which permits unrestricted non-commercial use, distribution, and reproduction in any medium, provided the original work is properly cited. can better reflect the relevant prevalence of mixed depression in clinical practice.

Some investigators have insisted that the mixed-features specifier must be replaced in agitated depression. ${ }^{6}$ The criteria of agitated depression also included psychomotor agitation, mood lability, and irritability, as well as racing thoughts, talkativeness, absence of retardation, weeping, and early insomnia. ${ }^{5}$ Those authors regarded agitation as the core symptom of mixed depression and bipolar disorder, and that patients with agitated depression do not respond well to antidepressants. ${ }^{6}$ The recently published Florida guideline also recommends including antipsychotics, mood stabilizer, and antidepressants (in that order) for patients in the mixed-features specifier of MDD?

Mixed depression is very important because the frequencies of suicide ideation, suicide attempts, comorbidities with anxiety disorder, and the use of antipsychotics and treatment cost are all greater in mixed depression than in MDD. ${ }^{8}$ Mixed depression is especially important in South Korea due to the relatively high rates of suicide attempts and acute stressful events. It is a very dynamic and highly competitive society due to the high population density and high education standards. Furthermore, Korean depressed patients exhibit a characteristic type of depression associated with anger. In addition, most of the depressed patients who present at my hospital with suicide attempts show major depressive episodes with psychomotor agitation, irritability, and mood lability like the agitated depression concept of Koukopoulos, and atypical antipsychotics have been very effective in these patients (unpublished data). It is therefore very important to include mixed depression when establishing suicide health-care policies in Korea.

It is particularly interesting that Italy-where the Koukopoulos group that strongly advocates the existence of agitated depression as a mixed type of depression and mainly performs clinical activities is based-has much in common with South Korea. Both countries are surrounded by sea on three sides and are at similar latitudes. Family ties are very strong in both societies, and they regard their family and bloodlines as very 
important. Koreans and Italians also exhibit common personality traits of urgency, passion, and kind heart, while the percapita GDP, GDP, population size, and geographic areas are all also similar. This suggests that agitated depression or mixed depression could reflect cultural and environmental factors. Psychosocial factors such as spousal cheating, fraud, debt, family death, and family conflicts play very important roles as causes of suicide in patients with mixed depression. Such factors might be very important contributing factors to mixed depression, and so the prevalence rate might be very high in Korea due to high levels of psychological stress. However, there has been very little research into this type of mixed depression worldwide.

It is fortunate that the clinical significance of agitated depression as a mixed depression has been acknowledged by some investigators. Future large-scale studies should investigate the diagnostic criteria of mixed depression and its treatments.

\section{Acknowledgments}

This article was supported by a grant from National Research Foundation of Korea (NRF), funded by Ministry of Education (NRF-2018R1D1 A1A02085847).

\section{REFERENCES}

1. Koukopoulos A, Sani G. DSM-5 criteria for depression with mixed features: a farewell to mixed depression. Acta Psychiatr Scand 2014;129:416.

2. Ghaemi SN. DSM-5 and the miracle that never happens. Acta Psychiatr Scand 2014;129:410-412.

3. Weibel S, Bertschy G. Mixed depression and DSM-5: a critical review. Encephale 2016;42:90-98.

4. Targum SD, Suppes T, Pendergrass JC, Lee S, Silva R, Cucchiaro J, et al. Major depressive disorder with subthreshold hypomania (mixed features): clinical characteristics of patients entered in a multiregional, placebo-controlled study. Prog Neuropsychopharmacol Biol Psychiatry 2016;68:9-14.

5. Sani G, Vohringer PA, Napoletano F, Holtzman NS, Dalley S, Girardi P, et al. Koukopoulos diagnostic criteria for mixed depression: a validation study. J Affect Disord 2014;164:14-18.

6. Koukopoulos A, Sani G, Koukopoulos AE, Manfredi G, Pacchiarotti I, Girardi P. Melancholia agitata and mixed depression. Acta Psychiatr Scand Suppl 2007;(433):50-57.

7. McIntyre RS, Suppes T, Tandon R, Ostacher M. Florida Best Practice Psychotherapeutic Medication Guidelines for Adults With Major Depressive Disorder. J Clin Psychiatry 2017;78:703-713.

8. McIntyre RS, Ng-Mak D, Chuang CC, Halpern R, Patel PA, Rajagopalan K, et al. Major depressive disorder with subthreshold hypomanic (mixed) features: a real-world assessment of treatment patterns and economic burden. J Affect Disord 2017;210:332-337. 